\title{
The implications of close binary stars for star-disk interactions
}

\author{
Robert D. Mathieu \\ Department of Astronomy, University of Wisconsin - Madison, WI 53706 USA \\ email: mathieu@astro.wisc.edu
}

\begin{abstract}
The presence of close $(\lesssim 0.1 \mathrm{AU})$ stellar companions must greatly alter the circumstellar environment of classical $\mathrm{T}$ Tauri stars, including severe truncation if not elimination of circumstellar disks. It is thus remarkable how little impact the presence of a close companion has on our observable diagnostics for accretion and outflow. Emission line shapes, degrees of continuum veiling, and spectral energy distributions are all indistinguishable between single classical $\mathrm{T}$ Tauri stars and classical T Tauri close binaries. Some of the most classical T Tauri stars that laid the foundation for our single-star accretion-disk paradigm have turned out to have close companions. Periodicities in spectral signatures are suggestive of the presence of accretion flows from circumbinary disks to the circumstellar regions; the subsequent flow of material through the circumstellar region to the stellar surface in the presence of a stellar magnetosphere is unstudied. Observations of stellar rotation distributions in close binaries suggest that inner disk regions may act to regulate stellar angular momentum.
\end{abstract}

Keywords. Binary stars, accretion streams, stellar rotation, UZ Tau E, DQ Tau.

\section{Introduction}

The presence of stellar† companions within protostellar disks raises fascinating dynamical questions regarding disk structures and accretion flows. At the heart of these questions is the expectation that such companions will clear gaps in accretion disks (e.g., Figure 1 of Artymowicz \& Lubow 1996), and that the balance of viscous and resonant forces at the inner edge of a circumbinary disk will prevent the flow of circumbinary material to the circumstellar disks and thus to the stellar surfaces.

The accretion disk morphology as a result of this clearing varies qualitatively with the binary semi-major axis. For the widest binaries $(\mathrm{a} \gg 100 \mathrm{AU})$ the morphology may be one or two circumstellar disks, likely with properties little different from disks around single stars. For binaries with separations of several tens of AU, the morphology may be one or two truncated circumstellar disks, with radii of $0.2-0.3$ times the semi-major axis. For close binaries ( $\lesssim 10 \mathrm{AU}$ ), the binary may be surrounded by a circumbinary disk and each star may have a truncated circumstellar disk. If the pre-main-sequence binary population is similar to that of the field (e.g., Duquennoy \& Mayor 1991), roughly 15\% of the population is binaries with semi-major axes of less than $1 \mathrm{AU}$. In fact, there is evidence that the pre-main-sequence binary frequency may be higher than in the field.

Here I wish to connect these issues to the subject of this symposium. Specifically, the question that I wish to put forward - but alas, not answer - is the following:

$\dagger$ Many of the points of this article also apply to massive planetary companions. At present, the issues are most directly explored in the context of stellar companions. 
Given that every indicator of circumstellar accretion and outflow survives in the face of severe (complete?) circumstellar disk truncation within the shortest period classical T Tauri binaries, what does this tell us about star-disk physics?

This question rests on the simple observation that classical T Tauri binary stars with periastron separations of as small as several stellar radii show the same diagnostics for accretion and outflow as do single classical T Tauri stars (or those in wide binaries). Put another way, considering only our observable diagnostics for accretion and outflow, we cannot distinguish those classical $\mathrm{T}$ Tauri stars that have very close stellar companions (except perhaps in periodic variation). Given that we expect such companions to greatly modify the properties of circumstellar disks, if not eliminate them completely, this comes as a possibly very significant surprise for our understanding of star-disk interactions.

\section{Evidence for accretion in young short-period binaries}

One of the most classical of all classical T Tauri stars is a case in point - UZ Tau E. Its properties include: high-amplitude, irregular photometric variability; $\mathrm{H} \alpha$ emission equivalent width in excess of $50 \AA$; strong ultraviolet excess; and heavily veiled spectra. Indeed, it has been classified as an eruptive variable with behavior akin to FU Ori stars (Herbig 1977). All of these phenomena are taken as evidence for accretion onto the stellar surface; Hartigan, Edwards \& Ghandour (1995) determine a large accretion rate of order $10^{-6} M_{\odot} \mathrm{yr}^{-1}$. UZ Tau $\mathrm{E}$ also shows signatures of outflows at $\mathrm{M} \approx 10^{-8} M_{\odot} \mathrm{yr}^{-1}$, and evidence for microjets (Hirth, Mundt \& Soif 1997). Finally, UZ Tau E shows large infrared excesses and the canonical Class II spectral energy distribution of a protostellar disk. Millimeter interferometry have shown UZ Tau E to be surrounded by a massive $\left(\approx 0.6 M_{\odot}\right)$ disk (Jensen, Koerner \& Mathieu 1996). In the present paradigm, all of these observational data would be interpreted as - indeed, could be taken as an archetype of a single star surrounded by an actively accreting disk that is continuous from very near the stellar surface to its outer radius of several hundred AU.

In fact, UZ Tau E is a very close binary with an orbital period of 19.13 days, an eccentricity of 0.33 , and a mass ratio of 0.30 (Prato et al. 2002; Martín et al. 2005; Jensen et al. 2007). Using orbital elements and an inclination estimate from Jensen et al. the periastron separation is only 0.10 AU. Using a photometric estimate for the primary stellar radius of $2 R_{\odot}$, this periastron separation is only of order 10 (primary) stellar radii.

While UZ Tau E is perhaps the most striking case in point, it is not alone. The classical T Tauri stars DQ Tau (periastron separation 0.05 AU; Mathieu et al. 1997; Basri, JohnsKrull \& Mathieu 1997) and AK Sco (0.08 AU; Alencar et al. 2003) also show accretion and outflow diagnostics, and are even closer binary systems. V4046 Sgr might also be included in this sample, albeit likely with a much lower accretion rate (e.g., Stempels \& Gahm 2004).

Again, the point of this brief summary is to emphasize that in these young binaries, all of our paradigmatic observables for accretion and outflow appear to be unaffected despite the presence of stellar companions passing within 10 stellar radii or less every 10-20 days. How might, for example, magnetospheric accretion from disk to stellar surface continue unaffected in such an enviroment?

\section{Accretion streams}

A decade ago Artymowicz \& Lubow (1996; AL) found in SPH simulations that for sufficiently warm and viscous circumbinary disks, material can flow across the cleared 
gaps as streams with mass transfer rates equivalent to those expected from continuous disks around single stars. This idea was further explored by Günther \& Kley (2002), who confirmed the presence of such streams using high-resolution numerical simulations (Figure 1).

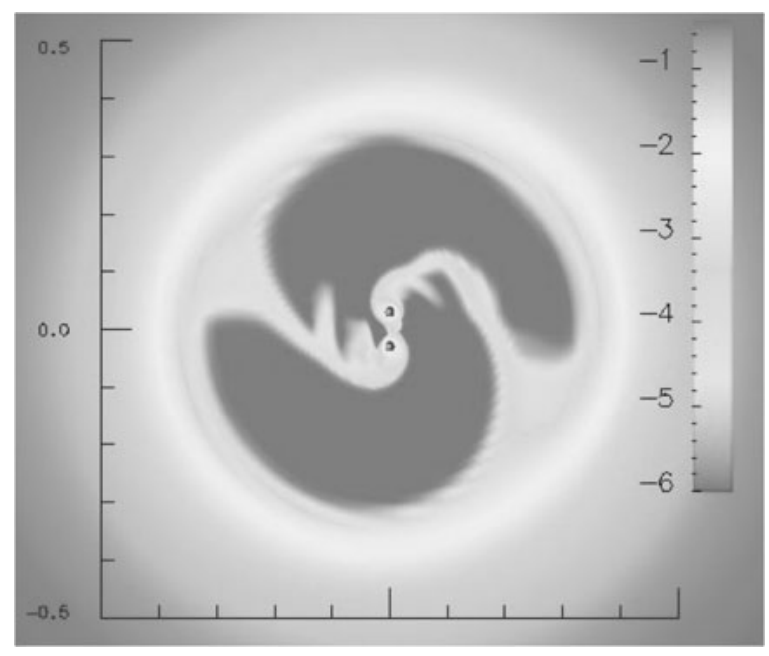

Figure 1. DQ Tau circumbinary disk after 85.5 orbital periods. The binary is shown at periastron, and the size of the stars reflects their actuall stellar radii. The length scales are in AU. (From Günther \& Kley 2002, where the figure can be found in color.)

Contemporaneous with AL, my collaborators and I were studying the classical T Tauri binary DQ Tau. This binary has an orbital period of 15.80 days, an eccentricity of 0.56 , and a mass ratio of unity (Mathieu et al. 1997; see also Mathieu 2001 for a summary discussion). The periastron separation is only $0.05 \mathrm{AU}$, just a few stellar radii (Figure 2). Nonetheless, the stellar spectra show diagnostics for both accretion and outflow (e.g., Figure 3), albeit at a lower accretion rate (e.g., $5 \times 10^{-8} M_{\odot} \mathrm{yr}^{-1}$ ) than for UZ Tau E.

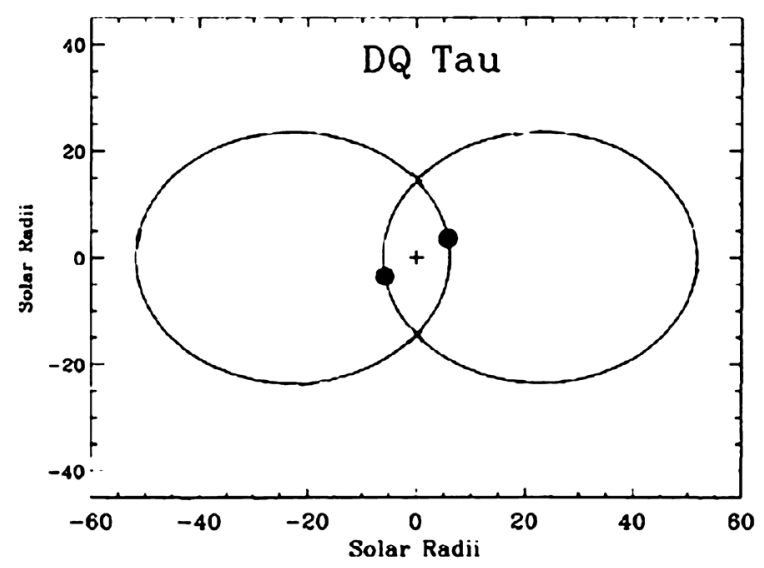

Figure 2. The orbit of the classical T Tauri binary DQ Tau, with the radii of the stars shown to scale. (Adapted from Basri, Johns-Krull \& Mathieu 1997.)

Importantly, photometric, veiling, and $\mathrm{H} \alpha$ observations all indicate enhanced accretion rates at periastron passage (Mathieu et al. 1997; Basri, Johns-Krull \& Mathieu 1997). Such pulsed accretion events were also found by AL in SPH calculations for a binary with 

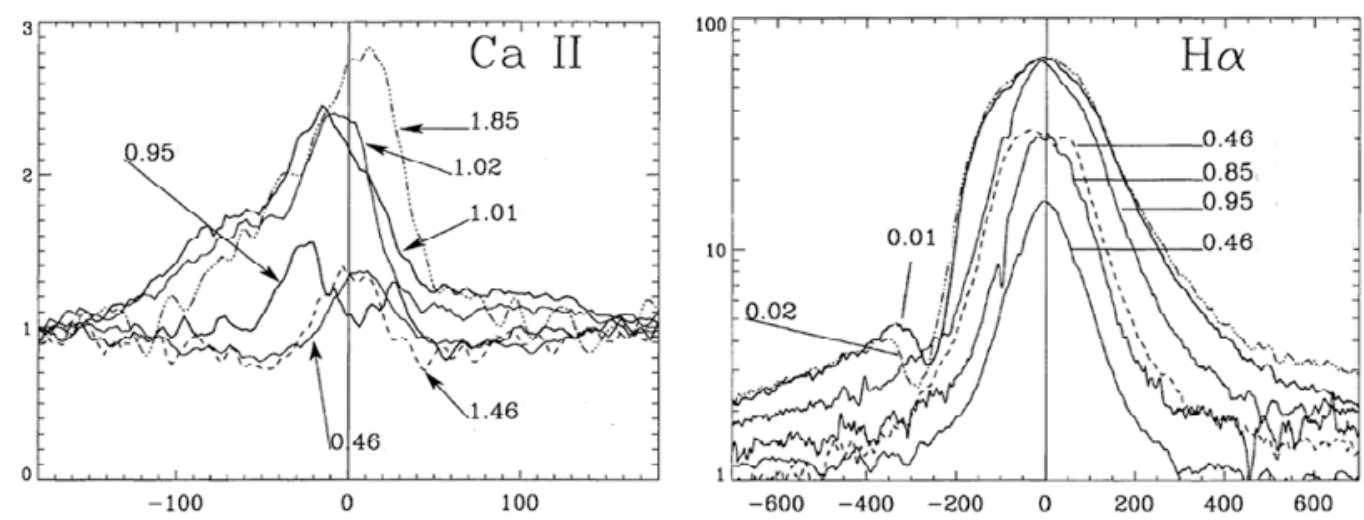

Figure 3. Time-series line profiles for DQ Tau. The labels are orbital counts and phases. During periastron the lines show outflow signatures; $\mathrm{H} \alpha$ develops blueshifted absorption features, and Ca II can display strong blueshifted emission. (From Basri, Johns-Krull \& Mathieu 1997. Note that the $\mathrm{H} \alpha$ scale is logarithmic.)

parameters very similar to DQ Tau, leading Mathieu et al. (1997) to suggest that the periodic accretion events of DQ Tau might be evidence for accretion streams. Later Carr, Mathieu \& Najita (2001) used observations of CO fundamental transitions to demonstrate the presence of $1200 \mathrm{~K}$ gaseous material located within the central few tenths of an AU of the binary system. The discovery of residual gas and dust within the regions expected to be cleared by the binary suggests a link between the circumbinary disk and the ongoing accretion at the stellar surfaces. That said, the relation of this material to an accretion stream is not yet established.

More recently, similar evidence for periodic accretion events has been searched for in the short-period classical T Tauri binaries AK Sco and UZ Tau E. Alencar et al. (2003) found no evidence of enhanced accretion near the periastron passage in AK Sco. They did find periodicity in the $\mathrm{H} \alpha$ emission with the orbital period, but its phasing is not evidently associated with theoretical predictions (AL, Günther \& Kley 2002). Jensen et al. (2007; see also Huerta, Hartigan, \& White, 2005) find the brightness of UZ Tau E to show significant random variability, but nonetheless to also show an overall periodic pattern with a broad peak spanning more than half the binary orbital period. Similar periodicity in the $\mathrm{H} \alpha$ emission is not evident, but the present data are sparse. They suggest that these variations are consistent with the theoretical predictions of accretion stream models.

Returning to the topic of this symposium, if accretion streams are present in these close binaries - or some other flow of circumbinary material to circumstellar regions the implications for star-disk interactions near the stellar surfaces are as yet unexplored. The theoretical simulations to date have not adequately investigated the hydrodynamics of the gas flows in the immediate vicinity of the stars. And indeed, magnetohydrodynamic simulations are likely needed. Recall that in DQ Tau the periastron separation is only $0.05 \mathrm{AU}$, just a few stellar radii. Circumbinary material crossing the gap does so within the domains of the stellar magnetospheres.

One clue to how the accreting material organizes itself may be the finding of Basri, Johns-Krull \& Mathieu (1997) that while the veiling of DQ Tau does increase at periastron, nonetheless some veiling - and by implication, some accretion - is present throughout the orbital cycle. (Note that this paper also presents an extensive discussion of the possible distribution of line-emitting gas around DQ Tau.) 
Another clue may lie in an interesting distinction between the spectral energy distributions (SEDs) of classical $\mathrm{T}$ Tauri close binaries and those weak-lined $\mathrm{T}$ Tauri stars that still show infrared excesses, as shown in Figure 4. The classical T Tauri close binaries show continuous Class II spectral energy distributions with minimal to no dips in emission at those wavelengths corresponding to the temperature of material missing from cleared regions. The spectral energy distributionss do not preclude such cleared regions (Mathieu et al. 1997; Alencar et al. 2003), but if gaps are present their signature is subtle. On the other hand, close weak-lined T Tauri binaries show substantial structure in their spectral energy distributions indicative of gaps (e.g., Jensen \& Mathieu 1997).
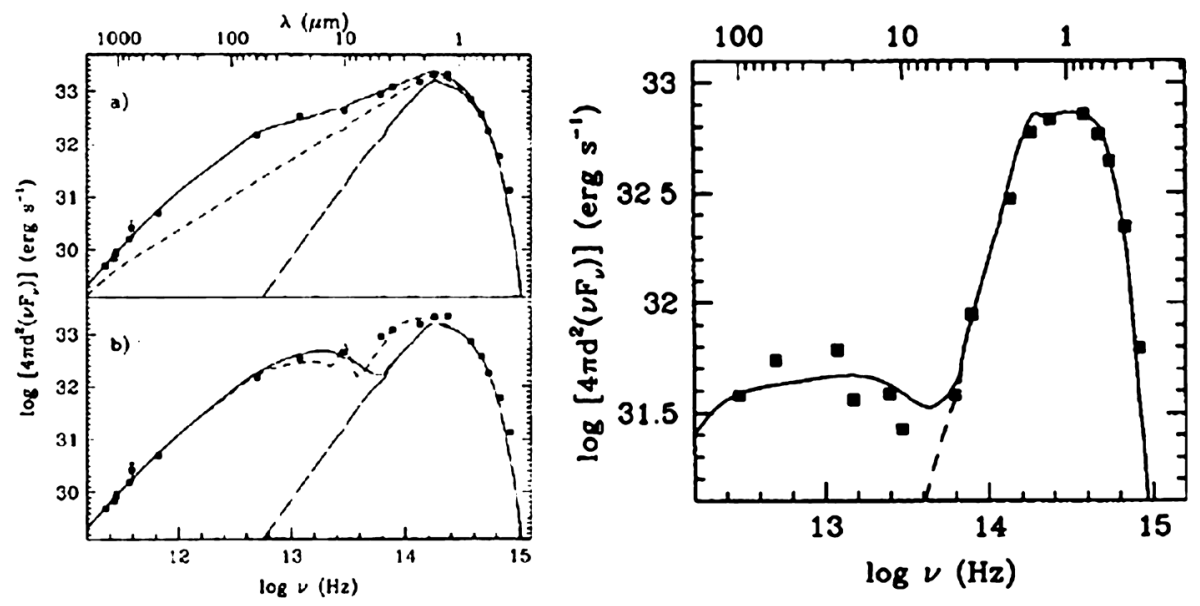

Figure 4. Left panel: The spectral energy distribution of DQ Tau, which is very similar to a canonical Class II spectral energy distribution from a continuous accretion disk. The long-dash line represents the stellar photosphere. The dashed curve in the upper panel represents a reprocessing disk, while the solid line is a best-fit active accretion disk, both continuous from the stellar surface. The solid line in the lower panel is an active accretion disk with a cleared central hole of radius $0.4 \mathrm{AU}$, while the dashed line represents a similar disk with warm optically thin dust in the central hole. (For more details, see Mathieu et al. 1997). Right panel: The spectral energy distribution of V4046 Sgr. Note the markedly different morphology from DQ Tau. The solid line represents an accretion disk with a central hole to the dynamically predicted outer radius of 0.072 AU. (From Jensen \& Mathieu 1997.)

In all likelihood, the actual distribution and flow of accreting material is more complex than we have considered. Basri, Johns-Krull \& Mathieu note evidence for "complex occultations, ejections, and absorptions near periastron, while the stars look rather placid away from each other." They conjecture that a "nimbus" of material may sit in the circumstellar environment of each star that could contribute to the continuous, lower level accretion. Whatever may be the reality, given this rather different accretion environment it remains all the more remarkable that the spectral signatures of these binaries are indistinguishable from single classical T Tauri stars.

Finally, a digression. While all of the discussion so far in this paper has been in the context of accretion disks, it is worth noting that at periastron it is possible that the stellar magnetospheres of both stars themselves interact. At the least we might expect to observe large flare events, such as seen in RS CVn binaries. Basri, Johns-Krull \& Mathieu (1997) consider whether such flares might be the proper interpretation of the photometric and spectroscopic events of DQ Tau at periastron, concluding that magnetospheric interactions were not likely to be the source of the photometric brightening or enhanced veiling and emission lines. Nonetheless, if the magnetospheres are as large 
as 5 stellar radii (as suggested by radio observations of some weak-lined T Tauri stars), then we might expect some interaction. Indeed, as expressed by these authors, it may be notable that DQ Tau is able to pass through roughly a third of its periastron passages without observed outbursts.

\section{Angular momentum regulation: insights from short-period binaries}

An increasing body of observational evidence suggests that early stellar rotation evolution is linked to the presence of accretion disks (see the review by Bouvier in this volume). Possibly the mechanism is linked to the coupling of the stellar magnetic field with the innermost region of the accretion disk, often referred to as "disk locking" (e.g., Herbst et al. 2007). While there has been animated discussion regarding the physics of such disk locking at this symposium, the observed association of observed disk diagnostics with longer stellar rotation period stands on its own.

Binary stars allow us to do "experiments" to determine which disk radii, if any, play a role in angular momentum regulation. Presuming that binary companions clear gaps in disks, binary stars allow us to remove regions of disks and examine the impact on stellar rotation. This approach was first taken by Bouvier, Rigaut \& Nadeau (1997), who looked for a correlation between $v \sin i$ and binary separation among solar-type stars in the 120 Myr old Pleiades open star cluster. Because their binary sample comprised angularly resolved systems they were able to only consider binaries with separations greater than $10 \mathrm{AU}$. They found no correlation of stellar rotation with binary separation in this separation range.

Later Patience et al. (2002) did a similar study in the 90 Myr cluster $\alpha$ Per, again using a sample of angularly resolved binaries with separations greater than $10 \mathrm{AU}$. They suggested the presence of an increase in $v \sin i$ for their four binaries with separations of less than $60 \mathrm{AU}$, with a confidence level of $98 \%$.

The magnetic disk locking mechanism, along with other proposed mechanisms for linking stellar angular momentum evolution to disks, couples the star to innermost regions of the disk, within tenths of an AU from the stellar surface. As such, the binaries expected to have the most direct impact on disk regulation are those with the shortest periods. Thus Meibom, Mathieu \& Stassun (2007) recently have repeated these experiments using the spectroscopic binary population among solar-type stars in the 150 Myr open cluster M35.

Specifically, they compared the rotation periods (measured directly through photometric periodicity) of single stars and of primary stars in spectroscopic binaries with semi-major axes of 0.1 AU to 5 AU. Binaries with semi-major axes shorter than $0.1 \mathrm{AU}$ may have primary rotation velocities modified by tidal synchronization (Meibom, Mathieu \& Stassun 2006). Primary stars of binaries with separations greater than 5 AU are not detectable with spectroscopic techniques, and thus are included among the "single" star sample. These latter binaries are also wide enough that they are not expected to clear the innermost regions of their circumstellar disks.

The outcome of this study is presented in Figure 5. The primary stars in the shorter period binaries rotate more rapidly than the combined sample of single stars and primary stars in wide binaries. The two distributions are distinct at the $99.9 \%$ confidence level.

The results of these three studies, and especially the last, are consistent with mechanisms for stellar angular momentum regulation linked to the innermost regions of accretion disks. That said, it is important to recognize the difference between correlation and causality. Given our almost complete lack of understanding about how such close 


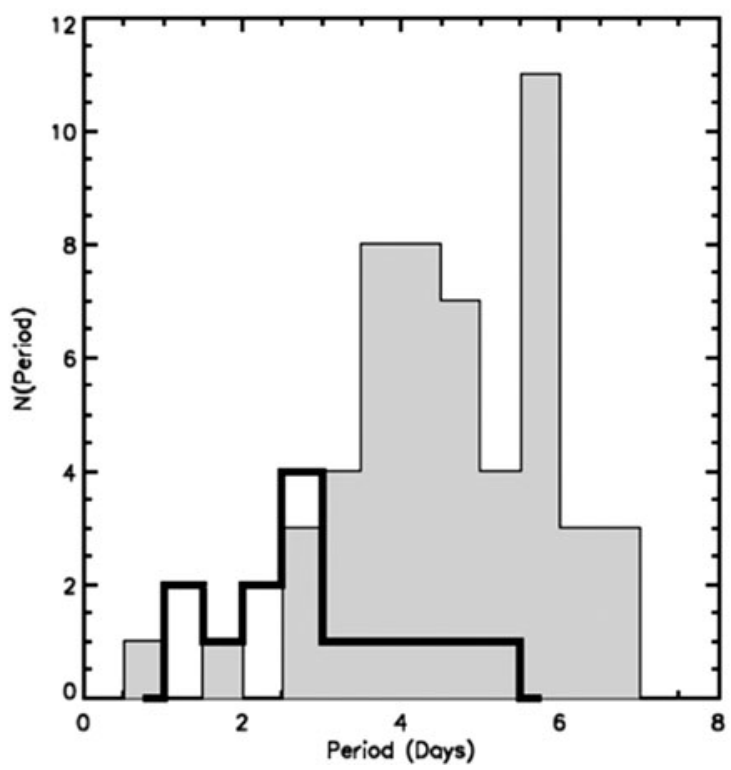

Figure 5. Rotation period distributions for single stars (grey histogram; $\left\langle P_{\text {rot }}\right\rangle=4.64$ days) and binary primary stars (solid; $\left\langle P_{r o t}\right\rangle=2.95$ days) in the $150 \mathrm{Myr}$ open cluster M35.

binaries form, we must at least entertain the conjecture that rapid stellar rotation and close binaries may be linked through the binary formation process itself.

\section{Summary}

In one sense, the dynamical interaction of orbiting stars with an accretion disk is one of the most dramatic star-disk interactions, with extensive impact on mass distribution over many AU. Here, however, we have focussed on the implications of close stellar companions for the accretion and outflow processes that have been the primary topic of this symposium.

Three essential findings merit emphasis in this summary:

1. At least $15 \%$ of $T$ Tauri stars are binaries with separations within $1 \mathrm{AU}$.

As observers and interpreters of observational data, we ignore this fact at our own peril. Interesting signatures and behaviors of a supposed single star, and interpreted in the context of single-star-disk interactions, may in fact be associated with the presence of a stellar companion, either through contribution to the combined light or through physical interaction.

2. The presence of close (e.g., $\lesssim 0.1 \mathrm{AU})$ companions does not change the classical $T$ Tauri signatures of accretion and outflow.

Given that we expect such companions to greatly modify the properties of circumstellar disks, if not eliminate them completely, it is remarkable how little impact the presence of a companion has on our standard diagnostics for accretion and outflow. Emission lines, continuum veiling, and spectral energy distributions are all indistinguishable between single classical T Tauri stars and classical T Tauri close binaries. 
The one property revealing classical $\mathrm{T}$ Tauri close binaries is occasional periodicities in these observable diagnostics commensurate with the orbital period. In the case of DQ Tau in particular, the presence of accretion events at periastron are suggestive of the presence of accretion streams from the circumbinary disk to the circumstellar regions.

The process by which the material in such streams reaches the stellar surfaces remains unknown. Given the very small periastron distances of only a few stellar radii, it is unlikely that we can simply transfer our current single-star picture of a circumstellar accretion disk truncated by the stellar magnetic field and from which material flows onto the stellar field lines to the star. Indeed given that the inner radius of the region cleared by the binary lies within the stellar magnetospheres, a circumstellar disk may not be part of the accretion process at all. The direct interplay of an accretion stream with a stellar magnetosphere needs to be explored.

3. Primary stars in young (150 Myr), short-period binaries rotate more rapidly than primary stars in wide binaries or single stars.

This finding is consistent with the regulation of stellar angular momentum by a mechanism associated with the inner regions of accretion disks, such as magnetic disk locking. It is also possible that this result reflects on the redistribution of angular momentum in the binary formation process itself.

The primary point of this paper is that our observable diagnostics for accretion and outflow associated with classical $\mathrm{T}$ Tauri stars are remarkably robust in the presence of very close stellar companions. Arguably, that these diagnostics are so robust is encouraging. Nonetheless, and being intentionally provocative, if our diagnostics are independent of the presence of a close companion that is assuredly greatly modifying the circumstellar environment, perhaps we might wonder to what extent we can in fact use these diagnostics to test models of star-disk interactions around even single classical T Tauri stars.

\section{Acknowledgements}

I would like to thank the organizers of this meeting for inviting me, and thereby finally convincing me that studying outflows in young close binaries is as intriguing as studying accretion! The work in this paper with which I have been associated has been done with many friends; I want to particularly acknowledge the contributions of my students Eric Jensen, Søren Meibom, and Keivan Stassun, from whom I have learned so much, and Gibor Basri, whose insights are present throughout this paper. I wish to gratefully acknowledge support of the IAU, and of the National Science Foundation over many years.

\section{References}

Alencar, S. H. P., Melo, C. H. F., Dullemond, C. P., Andersen, J., Batalha, C., Vaz, L. P. R., \& Mathieu, R. D. 2003, A\&\&A, 409, 1037

Artymowicz, P. \& Lubow, S. H. 1996 , ApJ, 467, L77

Basri, G., Johns-Krull, C. M., \& Mathieu, R. D. 1997, AJ, 114, 781

Bouvier, J., Rigaut, F., Nadeau, D. 1997, A\&SA, 323, 139

Carr, J. S., Mathieu, R. D., \& Najita, J. R. 2001, ApJ, 551, 454

Duquennoy, A. \& Mayor, M. 1991, A\&A, 248, 485 
Günther, R. \& Kley, W. 2002, A\&AA, 387, 550

Hartigan, P., Edwards, S. \& Ghandour, L. 1995 ApJ, 452, 736

Herbig, G. H. 1977 ApJ, 217, 693

Herbst, W., Eislöffel, J., Mundt, R., \& Scholz, A. 2007 In Protostars and Planets V, B. Reipurth, D. Jewitt, and K. Keil (eds.), University of Arizona Press, Tucson, 297

Hirth, G.A., Mundt, R. \& Soif, J. 1997 A\&AS, 126, 437

Huerta, M., Hartigan, P., \& White, R. J. 2005, AJ, 129, 985

Jensen, E. L. N., Dhital, S., Stassun, K. G., Patience, J.; Herbst, W. Walter, F. M., Simon, M., \& Basri, G. 2007, AJ, 134, 241

Jensen, E. L. N., Koerner, D. W.,\& Mathieu, R. D. 1996, AJ, 111, 2431

Jensen, E. L. N. \& Mathieu, R. D. 1997, AJ, 114, 301

Martín, E. L., Magazzú, A., Delfosse, X., \& Mathieu, R. D. 2005, A\&̋A, 429, 939

Mathieu, R. D., Stassun, K., Basri, G., Jensen, E. L. N., Johns-Krull, C. M., Valenti, J. A., \& Hartmann, L. W. 1997, AJ, 113, 1841

Mathieu, R. D. in The Formation of Binary Stars, Proceedings of IAU Symp. 200, Edited by Hans Zinnecker and Robert D. Mathieu, 2001, p. 419.

Meibom, S., Mathieu, R. D., \& Stassun, K. G. ApJ, in press

Meibom, S., Mathieu, R. D., \& Stassun, K. G. 2006, ApJ, 653, 621

Patience, J., Ghez, A. M., Reid, I. N., \& Matthews, K. 2002 AJ, 123, 1570

Prato, L., Simon, M., Mazeh, T., Zucker, S., \& McLean, I. S. 2002, ApJ, 579, L99

Stempels, H.C. \& Gahm, G.F. 2004, A\&A, 421, 1159

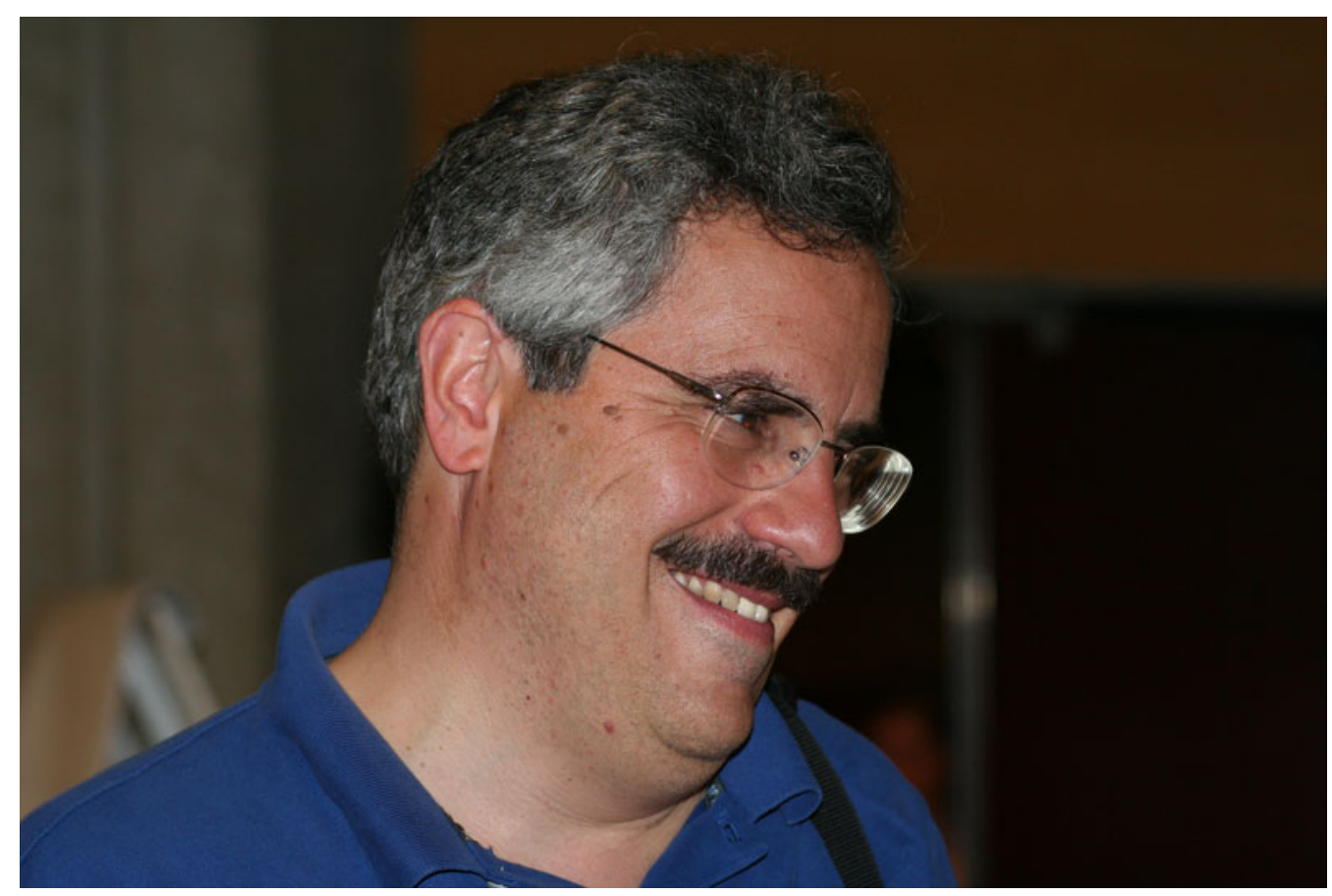



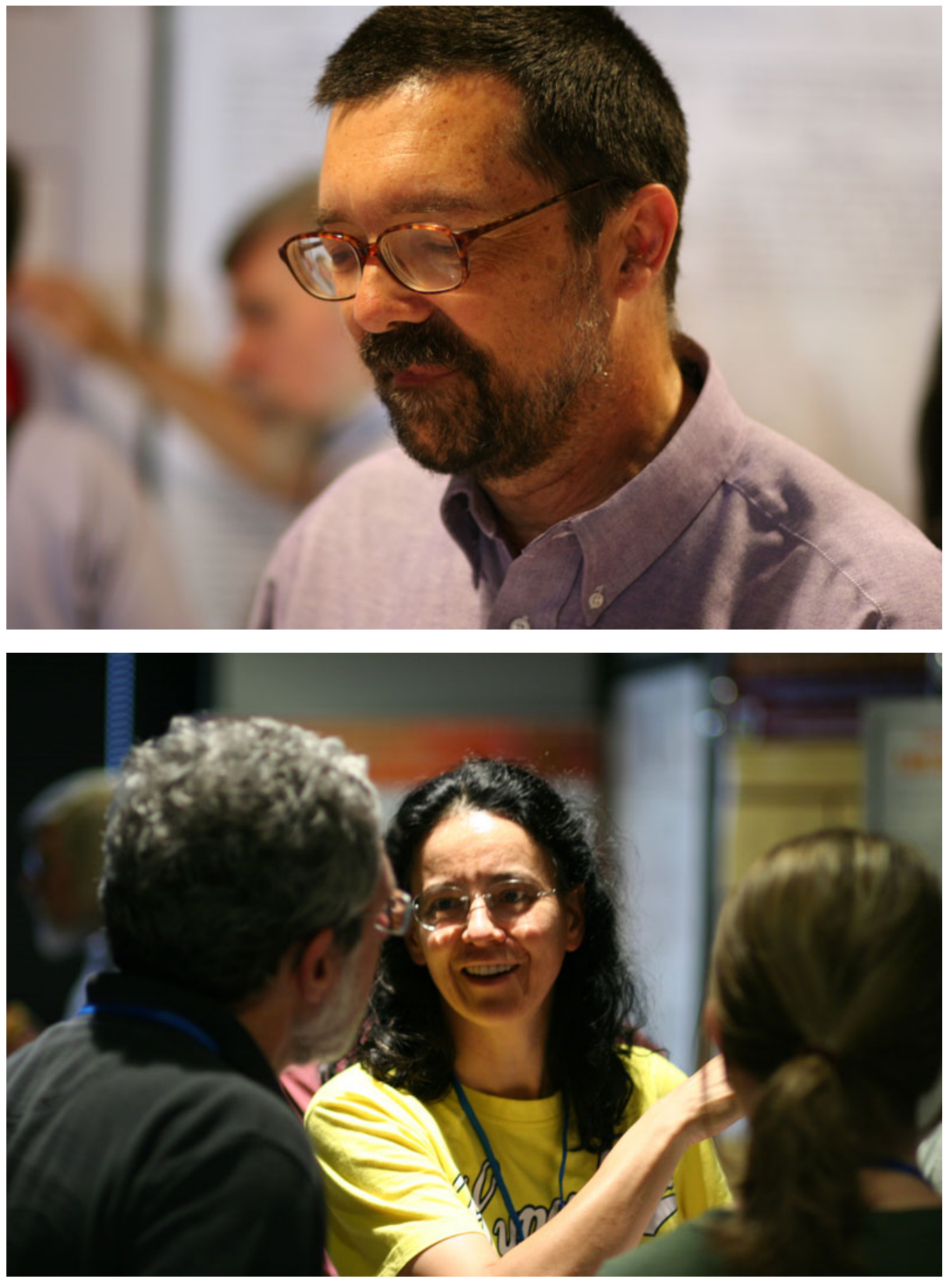